\title{
Perfil de pacientes internados por Acidente Vascular Cerebral em uma unidade de urgência e emergência em um hospital escola do Oeste do Paraná e a atuação do profissional fisioterapeuta nestes casos
}

\author{
Hospitalized patients profiles due to Cerebral Vascular Accident in a urgency and emergency unit \\ in a hospital school in West Parana and the performance of the physical therapist in these cases \\ Perfil de pacientes hospitalizados por Accidente Vascular Cerebral en una unidad de urgencia y \\ emergencia de un hospital universitario del Oeste de Paraná y la actuación del terapeuta físico en
} estos casos

Carla Lautenschleger Branco ORCID: https://orcid.org/0000-0003-4192-6099 Universidade Estadual do Oeste do Paraná, Brasil E-mail: carlalbranco@hotmail.com Juliana Hering Genske ORCID: https://orcid.org/0000-0001-6896-0903 Universidade Estadual do Oeste do Paraná, Brasil E-mail: juliana.hering@terra.com.br

Alana Caroline Bavaresco ORCID: https://orcid.org/0000-0002-0014-1183 Universidade Estadual do Oeste do Paraná, Brasil E-mail: alana-bavaresco@ hotmail.com

Caio Fernandes de Almeida ORCID: https://orcid.org/0000-0001-9108-5799 Universidade Estadual do Oeste do Paraná, Brasil E-mail: caiofernandes66@gmail.com

\begin{abstract}
Resumo
Objetivo: Fazer o levantamento dos casos atendidos pelo serviço de fisioterapia e quais foram as condutas utilizadas por esses profissionais e traçar o perfil de pacientes admitidos por Acidente Vascular Cerebral na unidade de emergência no ano de 2020. Metodologia: Estudo observacional, retrospectivo, transversal, de caráter quantitativo, analisando pacientes que deram entrada na sala de emergência do Hospital Universitário do Oeste do Paraná (HUOP), no período de um ano. A coleta de dados foi realizada utilizando-se uma ficha previamente elaborada contendo as variáveis do estudo; a análise foi realizada por meio de estatística descritiva simples. Resultados: Foram encontrados 1.020 prontuários, onde 17 prontuários foram excluídos. Dos prontuários analisados, 186 foram por AVC, com maior prevalência no AVC isquêmico (77,42\%), sexo masculino (51,08\%) e naqueles com idade $>60$ anos $(68,28 \%)$. Ocorreram 64 óbitos, sendo 48 com AVC isquêmico e 16 com AVC hemorrágico. 84,95\% dos pacientes receberam atendimento fisioterapêutico. Conclusão: Conclui-se que o atual estudo vai de acordo com demais pesquisas, com maior prevalência de casos de AVC isquêmico, além de maiores taxas de diagnósticos no sexo masculino e em idades acima de 60 anos. Quanto ao desfecho, a maioria dos pacientes receberam alta hospitalar, ocorrendo apenas 64 óbitos. Em relação a fisioterapia, foram principalmente empregadas condutas motoras e respiratórias associadas, e apenas $15,05 \%$ não receberam nenhum tipo de atendimento fisioterapêutico.
\end{abstract}

Palavras-chave: Acidente Vascular Cerebral; Fisioterapia; Prevalência; Serviços de atendimento de emergência.

\begin{abstract}
Objective: To survey the cases seen by the physiotherapy service and what procedures were used by these professionals and to trace the profile of patients admitted for cerebrovascular accident in the emergency unit in 2020. Methodology: Observational, retrospective study, cross-sectional, quantitative in nature, analyzing patients who were admitted to the emergency room of the Hospital Universitário do Oeste do Paraná (HUOP), in a period of one year. Data collection was performed using a previously elaborated form containing the study variables; an analysis was performed using simple descriptive statistics. Results: 1,020 records were found, where 17 were excluded. Of the frontal records, 186 were due to stroke, with a higher prevalence in ischemic stroke $(77.42 \%)$, male gender $(51.08 \%)$ and in those aged $>60$ years $(68.28 \%)$. There were 64 deaths, 48 with ischemic stroke and 16 with hemorrhagic stroke. $84.95 \%$ of patients received physical therapy care. Conclusion: It is concluded that the current study is in line
\end{abstract}


with other research, with a higher prevalence of ischemic stroke cases, in addition to higher rates of diagnoses in males and aged over 60 years. As for the outcome, most patients were discharged from the hospital, with only 64 deaths. Regarding physiotherapy, associated motor and respiratory behaviors were mainly used, and only $15.05 \%$ did not receive any type of physiotherapeutic care.

Keywords: Stroke; Physical therapy modalities; Prevalence; Emergency medical services.

\section{Resumen}

Objetivo: Relevar los casos atendidos por el servicio de fisioterapia y qué procedimientos fueron utilizados por estos profesionales y trazar el perfil de los pacientes ingresados por accidente cerebrovascular en la unidad de urgencias en 2020. Metodología: Estudio observacional, retrospectivo, transversal, cuantitativo en naturaleza, analizando los pacientes que fueron ingresados en el servicio de urgencias del Hospital Universitário do Oeste do Paraná (HUOP), en el plazo de un año. La recolección de datos se realizó mediante un formulario previamente elaborado que contiene las variables de estudio; Se realizó un análisis mediante estadística descriptiva simple. Resultados: Se encontraron 1.020 registros, de los cuales 17 fueron excluidos. De los registros frontales 186 se debieron a ictus, con mayor prevalencia en ictus isquémico $(77,42 \%)$, sexo masculino $(51,08 \%)$ y mayores de 60 años $(68,28 \%)$. Hubo 64 muertes, 48 por accidente cerebrovascular isquémico y 16 por accidente cerebrovascular hemorrágico. El 84,95\% de los pacientes recibió atención de fisioterapia. Conclusión: Se concluye que el presente estudio está en línea con otras investigaciones, con una mayor prevalencia de casos de ictus isquémico, además de mayores tasas de diagnósticos en varones y mayores de 60 años. En cuanto al resultado, la mayoría de los pacientes fueron dados de alta del hospital, con solo 64 muertes. Respecto a la fisioterapia, se utilizaron principalmente conductas motoras y respiratorias asociadas, y solo el 15,05\% no recibió ningún tipo de atención fisioterapéutica.

Palabras clave: Accidente Cerebrovascular; Fisioterapia; Prevalencia; Servicios médicos de urgencia.

\section{Introdução}

Em um hospital, o serviço de urgência e emergência é a principal porta de entrada para aqueles que necessitam de um atendimento imediato, por apresentarem alterações biofísicas e/ou psíquicas, apresentando ou não risco iminente de morte (Duarte et al., 2020).

As urgências caracterizam-se como aqueles atendimentos médicos que devem ser feitos com rapidez, por conta de um imprevisto de um agravo de saúde, porém, que não apresenta um risco de vida iminente. Já a emergência, é o surgimento ou aparecimento de algo, sendo aquela em que apresenta um risco iminente de vida, e deve ser tratado e diagnosticado nas primeiras horas após ser constatado (Coelho, 2019, Moura et al., 2018).

Segundo a Organização Mundial da Saúde, a segunda maior causa de morte no mundo é o Acidente Vascular Cerebral (AVC), a qual em 2016 foi responsável por cerca de 6,7 bilhões de óbitos, além disto, há uma tendência de ser responsável por 12,2\% dos óbitos previstos até o ano de 2030, mantendo-se na mesma posição (World Health Organization [WHO], 2018). No Brasil, segundo a Pesquisa Nacional de Saúde (PNS), no ano de 2013, estimou-se 2.231.000 casos de AVC e 568.000 casos de AVC apresentando deficiências graves (Bensenor et al., 2013).

O AVC é caracterizado por uma lesão neurológica, ou seja, uma série de eventos que afetam, de forma repentina, o sistema vascular intracraniano. Como causa, ocorre uma mudança no sistema vascular de alguma área do cérebro, devido ao rompimento ou obstrução de vasos sanguíneos, privando o suprimento sanguíneo neste local, levando a danos no sistema neural devido ao interrompimento do suprimento de nutrientes e oxigênio, ocorrendo assim, déficits neurológicos que podem ser focais ou globais. Pode ser dividido em dois grupos, de acordo com sua etiologia: AVC isquêmico e AVC hemorrágico (Sá et al., 2014, Rebouças et al., 2017).

O Acidente Vascular Cerebral Isquêmico (AVCi) é o mais comum e é classificado como aquele que interrompe o fluxo sanguíneo de uma área cerebral devido a um trombo, êmbolo ou até mesmo por uma compressão devido a uma massa tumoral, resultando assim em uma isquemia seguida, alguns minutos após, de um infarto, levando a um déficit neurológico focal e persistente (Rodrigues et al., 2017; Rolim \& Martins, 2011). Já o Acidente Vascular Cerebral Hemorrágico (AVCh), apesar de ser menos comum, é considerado o mais grave, e ocorre devido ao extravasamento sanguíneo em uma determinada 
área cerebral devido ao rompimento de um vaso, reduzindo assim a uma redução da efetividade da perfusão cerebral, lesando o tecido neurológico por anóxia (Barbosa et al., 2021; Sá et al., 2014; Lima et al., 2020).

Portanto, o AVC é um caso de emergência médica, e, como é de conhecimento geral, para os casos de urgência e emergência a assistência deve contar com profissionais de diversas áreas, ou seja, uma equipe multidisciplinar, contendo médicos, enfermeiros, técnicos de enfermagem, fisioterapeutas, entre outros, que devem trabalhar unidos para entregar o melhor tratamento possível a estes indivíduos (Almeida et al., 2017; Sociedade Brasileira de Doenças Cerebrovasculares [SBDCV], 2001).

O profissional fisioterapeuta foi inserido recentemente nas unidades de urgência e emergência, mais precisamente no ano de 2000 no Hospital Estadual do Grajaú em São Paulo, e sua presença torna-se indispensável e é de suma importância, principalmente quando se fala em pacientes com alterações cardiopulmonares que necessitam de oxigenoterapia ou ventilação mecânica. Além disto, a atuação do fisioterapeuta neste setor, juntamente com o restante da equipe, contribui para a redução do tempo de permanência do paciente na unidade e na melhora do quadro clínico, atuando na redução de índices e do tempo de intubação orotraqueal, ventilação mecânica invasiva e não invasiva, além do menor número de complicações (Almeida et al., 2017, Silva \& Santos, 2019).

Sendo assim, essa pesquisa se justifica pela escassez de estudos nacionais relacionados a ao perfil de pacientes com diagnóstico de AVC atendidos em unidades de emergência, bem como as características destes e a atuação do profissional fisioterapeuta neste contexto. Os dados coletados poderão auxiliar as equipes de saúde multiprofissionais que prestam serviço a essa população, subsidiando protocolos assistenciais, tanto em prevenção quanto em tratamento.

Diante disto, o objetivo do presente estudo foi fazer o levantamento dos casos atendidos pelo serviço de fisioterapia e quais foram as condutas utilizadas por esses profissionais e traçar o perfil de pacientes admitidos por AVC na unidade de emergência no ano de 2020.

\section{Metodologia}

O presente estudo classifica-se como observacional, transversal, documental e de caráter quantitativo (Vieira \& Hossne, 2021). Inicialmente, foi realizado um levantamento dos prontuários de pacientes admitidos na sala de emergência do Hospital Universitário do Oeste do Paraná (HUOP), no período de um ano, sendo de 1 de janeiro de 2020 até 31 de dezembro de 2020, através de busca por meio do software de gestão Tasy Rel. Em seguida, foram selecionados os pacientes internados neste período com diagnóstico principal de AVC, sendo do tipo isquêmico ou hemorrágico. Após, foi realizada a análise dos prontuários eletrônicos destes pacientes, através do software de gestão Tasy. A pesquisa foi iniciada após ser aprovada pelo Comitê de Ética em pesquisa da Universidade Estadual do Oeste do Paraná - UNIOESTE, com número de CAAE: 40327020.9.0000.0107.

Foram incluídos no estudo os pacientes internados na Sala de Emergência do HUOP com o diagnóstico principal de AVC isquêmico ou AVC hemorrágico de ambos os sexos e sem limites de idade. Foram excluídos aqueles pacientes que não ficaram internados na sala de emergência e aqueles internados por outras causas, como politraumas, tentativas de suicídio, doenças cardíacas, alterações pulmonares, entre outros.

Como instrumento de avaliação foi utilizada uma ficha previamente elaborada (Apêndice A), contemplando: dados de identificação (nome do paciente e número de atendimento), dados sociodemográficos (idade; sexo; naturalidade; estado civil; raça e escolaridade) e dados relacionados ao atendimento/internamento durante o período na sala de emergência (diagnóstico; se necessitou de oxigenoterapia; se chegou intubado ou necessitou da conduta durante o período de permanência na sala de emergência; uso de ventilação mecânica invasiva; se teve parada cardiorrespiratória e o tempo de permanência no local), destino do paciente após permanência no local (UTI; enfermaria; transferência para outro hospital; alta hospitalar ou óbito), 
destino final do paciente (alta hospitalar; óbito ou transferência hospitalar) e, por fim, dados sobre o atendimento fisioterapêutico (se recebeu atendimento; quantos atendimentos recebeu e condutas gerais).

Foi realizada uma busca ativa em dados do prontuário eletrônico do paciente, analisando detalhadamente os relatórios dos profissionais (médicos, enfermeiros, técnicos de enfermagem e fisioterapeutas) durante o período de internamento, a fim de coletar os dados do internamento, do atendimento fisioterapêutico e do desfecho do paciente, com auxílio da ficha descrita anteriormente.

Todos os dados coletados foram tabulados em uma planilha do Programa Excel 16.0 (Microsoft $囚$ ). A análise dos dados foi realizada por meio de estatística descritiva simples (média, desvio padrão, frequência absoluta e relativa).

\section{Resultados e Discussão}

O estudo analisou dados de pacientes internados no Hospital Universitário do Oeste do Paraná (HUOP) em CascavelPR., o qual trata-se de um Hospital Escola e centro de referência em saúde que presta serviço à população desta cidade e de todos os municípios da região Oeste e Sudeste do Estado do Paraná. Apresenta 100\% dos seus leitos e atendimentos destinados ao Sistema Único de Saúde (SUS), abrangendo 4 regionais, para aproximadamente 2 milhões de habitantes (Universidade Estadual do Oeste do Paraná, 2020). Conta com uma unidade de Pronto Socorro, a qual apresenta leitos de estabilização (Sala de Emergência), que atualmente também serve para o internamento de pacientes que aguardam vagas de Unidade de Terapia Intensiva (UTI), além das vagas de observação, para aqueles que aguardam leitos em enfermarias ou outras unidades (Baron, 2020).

O número total de internações na sala de emergência do Hospital Universitário do Oeste do Paraná, no período de abrangência da referida pesquisa, foi de 1.020 pacientes, segundo o software de gestão Tasy. Destes, 17 prontuários foram excluídos devido aos pacientes não terem sido realmente admitidos no local. Dos prontuários analisados, 186 (18,54\%) foram por AVC, nos quais 144 (77,42\%) pacientes foram vítimas de AVC isquêmico e 42 (22,58\%) AVC hemorrágico (Tabela 1).

De acordo com a literatura, sabe-se que o AVC ocorre devido a disfunções no fluxo sanguíneo cerebral e pode ser classificado como hemorrágico ou isquêmico, onde o AVC isquêmico é mais frequente e apresenta maior morbidade, ocorrendo em aproximadamente $80 \%$ dos casos. Já o AVC hemorrágico é mais raro, porém resulta em maior mortalidade (Lopes et al., 2016). Devido ao AVC isquêmico apresentar maior prevalência em geral, destacam-se seus fatores de risco, que podem ser classificados em modificáveis e não modificáveis. Os principais fatores de risco modificáveis são a hipertensão arterial sistêmica, fibrilação atrial, diabetes mellitus, dislipidemias e tabagismo, os quais são de conhecimento de que são encontrados em boa parte da população mundial nos dias atuais, inclusive a associação dos mesmos, auxiliando assim no aumento de casos de AVC isquêmico. Já os fatores de risco não modificáveis são: sexo, idade, hereditariedade, e localização geográfica, onde de acordo com isto, é encontrado o maior risco em pessoas do sexo masculino, e também, as chances de ocorrência do AVC isquêmico duplicam após os 55 anos de idade (Rodrigues et al., 2017).

Sendo assim, os resultados encontrados relacionados a maior prevalência de AVC isquêmico nos casos de pacientes internados com diagnóstico de AVC corroboram com a literatura e com os resultados encontrados em outros estudos nacionais dos autores Sá et al. (2014), Lima et al. (2015), Silva et al. (2018), onde foi verificado uma prevalência de AVC isquêmico de $78,4 \% ; 74,0 \%$ e 72,5\% respectivamente. Além disto, tal prevalência também foi encontrada em estudos internacionais, como o de Dabilgou et al. (2020), com 61\% de prevalência no AVC isquêmico. O estudo de Santos e Waters (2020), que se trata de uma revisão integrativa da literatura, também encontrou maior prevalência no AVC isquêmico, onde a incidência variou de $70,4 \%$ a $93,9 \%$ nos artigos analisados pelos pesquisadores.

Em geral, conforme demonstrado na Tabela 1, a idade média foi de $66,95( \pm 14,19)$ anos, tendo como idade mínima de 22 anos e idade máxima de 93 anos. Quanto ao AVC isquêmico, a média de idade foi de 67,81 $( \pm 13,41)$ anos, com variação de 
25 a 93 anos. Já em relação ao AVC hemorrágico, foi verificada uma idade média de 64,00 $( \pm 16,42)$ anos, variando de 22 a 89 anos. Em relação ao sexo, $51,08 \%$ eram do sexo masculino e $48,92 \%$ do sexo feminino, tal predominância também foi encontrada quando analisados separadamente, com 50,96\% de homens no AVC isquêmico e 52,38\% no AVC hemorrágico. No estudo de Mourão et al. (2017), foi encontrada uma idade média semelhante nos pacientes de AVC, com uma média de 64,3 anos, onde o mínimo era de 33 anos e o máximo de 93 anos, porém, o estudo não encontrou diferença significativa entre os sexos. Já Silva, Cobo, Foss, Vale e Cavenaghi (2021) encontraram uma média de 66,25 anos, com 56,04\% dos casos em homens. Percebe-se que a maior prevalência ocorre na população idosa e no sexo masculino, podendo ser devido ao motivo de que o aumento de idade é um fator de risco não-modificável, aumentando a vulnerabilidade nestes casos, e, no caso do sexo, pode estar associado a maior prevalência de Hipertensão Arterial Sistêmica em homens (Almeida \& Viana, 2018). Além disto, alguns estudos relatam que a maior prevalência de casos de AVC no sexo masculino se deve-se ao fato de que os homens têm maior propensão ao alcoolismo e tabagismo, os quais são fatores de risco relevantes para as doenças cardiovasculares e metabólicas (Santana et al., 2018).

Quanto à raça, a prevalência total foi de brancos, com 66,67\%, assim como quando analisados separadamente, onde a predominância de brancos foi de $67,36 \%$ no AVC isquêmico e $64,29 \%$ no AVC hemorrágico (Tabela 1). No estudo de Schmidt et al. (2019), 12,1\% dos pacientes com diagnóstico de AVC, com porcentagem por mil pessoas, eram da cor branca na região Sul do Brasil, região da qual também foi realizado o presente estudo. Também foi verificado no estudo citado, que tal prevalência também foi encontrada nas regiões Sudeste $(22,2 \%)$. Nas regiões Norte, Nordeste e Centro-Oeste, a maior porcentagem foi em pardos. No Brasil, em geral, a prevalência encontrada por Schmidt et al. (2019) foi de 48,2\% brancos, entrando em acordo com o atual estudo. No entanto, é de conhecimento que o presente estudo foi realizado na região Sul do Brasil, que possui maior predominância de brancos, e, o estudo de Schmidt et al. (2019) considerou todas as regiões do Brasil na prevalência.

Em relação à escolaridade (Tabela 1), em geral, a maior prevalência no presente estudo foi daqueles com ensino fundamental, com 33,33\%, porém em 39,78\% dos prontuários não foi encontrada a informação sobre esta variável. Tal resultado entra em desacordo com os resultados trazidos pela Pesquisa Nacional de Saúde, realizada pela Fundação Instituto Brasileiro de Geografia (IBGE, 2013), onde 69,8\% dos pacientes não possuíam instrução ou possuíam ensino fundamental incompleto. Porém, tal comparação torna-se dificultada, por conta da grande porcentagem não encontrada da referida informação na atual pesquisa. O baixo nível de escolaridade pode ser um fator de risco importante pois apresentam maior dificuldade em aderir aos tratamentos, no acesso a informações e também aos meios de tratamento, podendo ser um fator de risco para uma maior dependência do paciente em suas atividades (Fernandes et al., 2012, Schmidt et al., 2019). Por fim, quanto ao estado civil, a predominância foi de casados, com 44,09\% dos casos, entrando em acordo com o estudo de Dabilgou et al. (2020), onde também foi verificada uma maior prevalência de casados, com 83,8\% dos casos. 
Tabela 1. Descrição dos tipos de AVC e de características sociodemográficas de pacientes atendidos na sala de emergência do Hospital Universitário do Oeste do Paraná $(\mathrm{n}=186)$.

\begin{tabular}{|c|c|c|}
\hline Variável & $\mathbf{N}$ & $\%$ \\
\hline \multicolumn{3}{|l|}{ Tipo de AVC } \\
\hline Isquêmico & 144 & $77,42 \%$ \\
\hline Hemorrágico & 42 & $22,58 \%$ \\
\hline \multicolumn{3}{|l|}{ Idade } \\
\hline$<45$ anos & 11 & $5,91 \%$ \\
\hline $45-60$ anos & 48 & $25,81 \%$ \\
\hline$>60$ anos & 127 & $68,28 \%$ \\
\hline \multicolumn{3}{|l|}{ Sexo } \\
\hline Masculino & 95 & $51,08 \%$ \\
\hline Feminino & 91 & $48,92 \%$ \\
\hline \multicolumn{3}{|l|}{ Nacionalidade } \\
\hline Brasileiro & 182 & $97,85 \%$ \\
\hline Haitiano & 4 & $2,15 \%$ \\
\hline \multicolumn{3}{|l|}{ Escolaridade } \\
\hline Não informado & 72 & $39,78 \%$ \\
\hline Fundamental & 62 & $33,33 \%$ \\
\hline Médio & 26 & $13,98 \%$ \\
\hline Superior & 1 & $0,54 \%$ \\
\hline Analfabeto & 23 & $12,37 \%$ \\
\hline \multicolumn{3}{|l|}{ Cor/Raça } \\
\hline Branco & 124 & $66,67 \%$ \\
\hline Negro & 7 & $3,76 \%$ \\
\hline Pardo & 44 & $23,66 \%$ \\
\hline Não informado & 11 & $5,91 \%$ \\
\hline \multicolumn{3}{|l|}{ Estado civil } \\
\hline Solteiro & 21 & $11,29 \%$ \\
\hline Casado & 82 & $44,09 \%$ \\
\hline Divorciado & 15 & $8,06 \%$ \\
\hline Viúvo & 35 & $18,82 \%$ \\
\hline Amasiado & 9 & $4,84 \%$ \\
\hline Não informado & 24 & $12,90 \%$ \\
\hline
\end{tabular}

Fonte: Autoria Própria (2022).

Na Tabela 2 pode-se observar que apenas 22,04\% dos pacientes fizeram uso de oxigenoterapia. Já em relação a intubação orotraqueal (IOT), apenas $11,83 \%$ foram submetidos a intubação orotraqueal durante o período de internamento na sala de emergência e 33,33\% já foram admitidos com tubo orotraqueal. Os demais pacientes, 54,84\%, não necessitaram de intubação orotraqueal e apenas 2 indivíduos $(1,08 \%)$ foram submetidos a traqueostomia no período de internamento na sala de emergência.

No estudo de Aquino (2019), em 210 pacientes com AVC, 42 (20\%) necessitaram de intubação orotraqueal, ou seja, fizeram uso de VMI. Já Silva et al. (2014) realizaram um estudo em uma Unidade de AVC do Hospital Risoleta Tolentino Neves, na cidade de Belo Horizonte, com pacientes de idade igual ou superior a 60 anos, onde foram incluídos 349 pacientes. Destes, apenas $9(2,5 \%)$ necessitaram de ventilação mecânica invasiva e apenas 1 fez uso de ventilação mecânica não invasiva. 
Os resultados destes estudos são relativamente baixos quando comparados aos do atual estudo (Tabela 2), onde 84 indivíduos (45,16\%) fizeram uso de ventilação mecânica invasiva (VMI), sendo que o AVC hemorrágico apresentou maior prevalência do uso de VMI, com 66,67\% dos pacientes. Já no AVC isquêmico, foi verificada uma prevalência de 38,19\% dos indivíduos. Tal resultado pode estar relacionado ao perfil dos pacientes internados no HUOP, um Hospital Escola e Público, onde os recursos são mais escassos quando comparados aos estudos citados, pois a pesquisa de Aquino (2019) se passa em um hospital que conta com atendimentos pelo Sistema Único de Saúde e também atendimentos particulares, e apresentou um limite de idade de 18 anos. No entanto a maior diferença foi encontrada quando comparado ao estudo de Silva et al. (2014), que além de apresentar um limite de idade no estudo (pacientes acima de 60 anos), se passa em uma unidade especializada em AVC, apresentando uma equipe multidisciplinar e um processo de reabilitação especifico para a doença, com uma boa taxa de eficácia, que pode estar relacionado a redução e prevenção de complicações advindas da lesão cerebrovascular destes pacientes, otimizando o tratamento oferecidos a estes indivíduos.

Os pacientes permaneceram em média 2,43 $( \pm 2,22)$ dias na sala de emergência, onde o mínimo foi de 1 dia e máximo de 18 dias. Quanto ao destino do paciente após o internamento no local (Tabela 2), verificou-se que 99 (53,23\%) foram encaminhados para a enfermaria, $66(33,33 \%)$ para a UTI, 6 (3,23\%) foram transferidos para outra unidade hospitalar e 19 $(10,22 \%)$ entraram em óbito ainda no período de sala de emergência. Em relação ao desfecho hospitalar, no total, 64 (34,41\%) foram a óbito e $116(62,37 \%)$ receberam alta hospitalar. Quanto à transferência hospitalar no desfecho, manteve-se os 3,23\%.

Já no estudo de Radanovic (2000), 17,3\% pacientes foram transferidos para outra unidade hospitalar por falta de leito especializado (por exemplo, intervenção neurocirúrgica) ou porque o paciente tinha acesso a atendimentos com maior grau de conforto. Dos pacientes que não foram transferidos, $46,5 \%$ foram transferidos para enfermarias ou UTI e 53,5\% permaneceram no setor de emergência. Estes pacientes permaneceram no hospital por uma média de 4,11 dias, onde a maior média foi para os pacientes de AVC hemorrágico com uma média de 4,25 dias. Inicialmente, é necessário se atentar que o estudo citado trata-se de um estudo relativamente antigo quanto comparado ao atual estudo, podendo tal questão interferir na comparação, visto que com o passar do tempo, as equipes e instituições apresentam melhorias gradativamente. Além disto, o estudo citado relata que um dos motivos de transferência hospitalar é devido ao tratamento mais especializado, como por exemplo a intervenção neurocirúrgica, onde o hospital do atual estudo apresenta tal intervenção, podendo estar relacionado a menos transferências hospitalares. No demais, o estudo citado não apresenta demais informações quanto aos motivos que possam justificar o maior tempo de permanência dos pacientes no setor de emergência.

Alhazzani et al. (2018) estudou os casos de AVC hospitalizados pela primeira vez na região de Aseer, na Arábia Saudita, no período de um ano, onde ocorreu um tempo médio de internação de 10,67 $( \pm 17,38)$ dias e uma mediana de 7 dias, apresentando 121 mortes, ou seja, uma taxa de letalidade de 9,7\%, a qual é inferior à do atual estudo. Já em um estudo nacional, Almeida e Vianna (2018), apresentaram um tempo médio de internação de 9,5 ( $\pm 12,2$ dias), onde variou de 1 a 112 dias, apresentando uma taxa de mortalidade de 12,7\%. Deve-se levar em conta que a média de dias de internamento no atual estudo foi apenas durante o período de sala de emergência, enquanto os estudos citados tratam de um período de internamento hospitalar em geral. Em relação a mortalidade, o resultado pode se dar devido ao hospital do estudo citado se tratar de um hospital que atende tanto pelo Sistema Único de saúde quanto convenio e particular, sendo um hospital considerado referência e alta complexidade em Neurologia. Outros fatores foram comuns aos do atual estudo, sendo assim, apesar do HUOP também ser referência na região do estudo, deve-se levar em conta que a presente pesquisa se passa em um hospital de ensino, onde ocorre um número mais elevado de abordagens dos pacientes por estudantes e profissionais, uma quantidade relativamente grande de leitos e admissões hospitalares, além do fato de ser permitido acompanhantes nas enfermarias, predispondo a infecções que podem levar ao aumento da mortalidade. É de interesse investigar futuramente outras possibilidades relacionadas 
ao atendimento no hospital, para que seja possível compreender a discrepância entre as taxas de mortalidade no HUOP e outras unidades hospitalares que apresentam menor número de óbitos e complicações.

Tabela 2. Descrição de dados clínicos do paciente com AVC durante o internamento na sala de emergência do Hospital Universitário do Oeste do Paraná $(\mathrm{n}=186)$.

\begin{tabular}{|c|c|c|}
\hline Variável & $\mathbf{N}$ & $\%$ \\
\hline \multicolumn{3}{|l|}{ Oxigenoterapia } \\
\hline Não & 145 & $77,96 \%$ \\
\hline Sim & 41 & $22,04 \%$ \\
\hline \multicolumn{3}{|l|}{ Intubação orotraqueal (IOT) } \\
\hline Admitido com tubo orotraqueal & 62 & $33,33 \%$ \\
\hline Submetido a IOT durante internamento na sala de emergência & 22 & $11,83 \%$ \\
\hline \multicolumn{3}{|c|}{ Não necessitou de IOT no período de internamento na sala de emergência } \\
\hline & 102 & $54,84 \%$ \\
\hline \multicolumn{3}{|l|}{ Traqueostomia (TQT) } \\
\hline Realizado TQT durante internamento na sala de emergência & 2 & $1,08 \%$ \\
\hline Não realizado/não necessitou & 184 & $98.92 \% \%$ \\
\hline \multicolumn{3}{|l|}{ Uso de ventilação mecânica invasiva (VMI) } \\
\hline Sim & 84 & $45,16 \%$ \\
\hline Não & 102 & $54,84 \%$ \\
\hline \multicolumn{3}{|l|}{ Destino do paciente após saída da sala de emergência } \\
\hline UTI & 62 & $33,33 \%$ \\
\hline Enfermaria & 99 & $53,23 \%$ \\
\hline Óbito (ainda na sala de emergência) & 19 & $10,22 \%$ \\
\hline Transferência hospitalar & 6 & $3,23 \%$ \\
\hline \multicolumn{3}{|l|}{ Desfecho hospitalar do paciente } \\
\hline Alta & 116 & $62,37 \%$ \\
\hline Óbito & 64 & $34,41 \%$ \\
\hline Transferência hospitalar & 6 & $3,23 \%$ \\
\hline
\end{tabular}

Fonte: Autoria Própria (2022).

Santos et al. (2020) compararam o número de óbitos entre os anos de 2008 e 2018 em pacientes hospitalizados devido a AVC no estado do Paraná, o mesmo onde localiza-se o hospital do presente estudo. A pesquisa foi realizada pelo sistema DATASUS, onde verificou-se um total de 185.333 óbitos na região Sul do Brasil, sendo 69.248 no estado do Paraná, correspondendo a 37,36\% dos casos da região Sul. O estudo ainda traz que o ano com maior número de óbitos notificados foi o ano de 2016, com 6.600 óbitos.

Conforme já relatado, 64 pacientes $(34,41 \%)$ foram a óbito no presente estudo e quando analisados separadamente, o AVC hemorrágico apresentou uma mortalidade de 38,09\% (16 pacientes) e o AVC isquêmico uma mortalidade de 33,33\% (48 pacientes). Em ambos os tipos a maior mortalidade ocorreu tardiamente, ou seja, fora da sala de emergência (Figura 1).

No estudo de Santana et al. (2018), foi realizada uma análise dos dados epidemiológicos de AVC no Brasil de acordo com o estudo Global Burden of Disease (GBD) em 2016, onde foi encontrado uma quantia de 107.258 óbitos pela doença, onde a maioria era do sexo masculino (56.782 óbitos, 52,9\%) e naqueles com mais de 70 anos de idade (64.582 mortes, 60,2\%). Foi observado também, que o AVC isquêmico apresentou a maior prevalência de mortes (66.261 óbitos, 61,8\%). Na 
pesquisa de Sá et al. (2014), relataram 27 casos de óbitos, em uma amostra de 125 pacientes, ou seja, uma porcentagem de 21,6\%. Destes óbitos citados na pesquisa, 20 (74,1\%) foram naqueles com diagnóstico de AVC isquêmico. Ambos os estudos referenciados não corraboraram com o resultado do atual estudo, onde a maior prevalência foi nos casos de AVC isquêmico.

Figueiredo et al. (2020) realizou um estudo com uma amostra de 1367 indivíduos, sendo 1044 casos de AVC isquêmico e 323 casos de AVC hemorrágico. Os autores realizaram uma análise de sobrevivência em uma subamostra com 311 pacientes, onde foi verificado 166 óbitos, sendo 90 óbitos (54,2\%) por AVC hemorrágico, contra 76 (45,8\%) por AVC isquêmico. Sendo assim, a pesquisa observou uma menor probabilidade de sobrevivência nos casos de AVC hemorrágico, com cerca de 1,37 vezes (IC 95,0\%; 1,01-1,86) quando comparado aos casos de AVC isquêmico. Quando pensamos nos casos analisados separadamente, o atual estudo também apresentou maior prevalência de óbitos nos casos de AVC hemorrágico. O AVC hemorrágico ocorre devido a uma ruptura em um vaso cerebral, levando ao extravasamento de sangue dentro do cérebro ou na superfície entra a meninge e o cérebro, e, em geral, é responsável por 15\% dos casos (Silva et al., 2021). Ainda de acordo com Figueiredo et al. (2020), o AVC hemorrágico apresenta por si só um fator de risco independente para a mortalidade, de acordo com os mecanismos fisiopatológicos subjacentes a ambos os tipos de AVC, onde o tipo isquêmico ocorre devido à uma interrupção ou diminuição do fluxo de sangue no cérebro, podendo ser decorrente de uma embolia, trombose ou ateromatose, já o AVC hemorrágico ocorre devido a um extravasamento de sangue para fora da artéria, advindo de uma rutura de aneurisma, dilatação arterial ou hipertensão, sendo assim o estudo traz que o AVC hemorrágico apresenta, em média, cerca de 1,37 vezes menos probabilidade de sobrevivência, estando assim, associado a maior índice de gravidade e mortalidade.

Figura 1. Número de óbitos relacionados aos tipos de AVC.

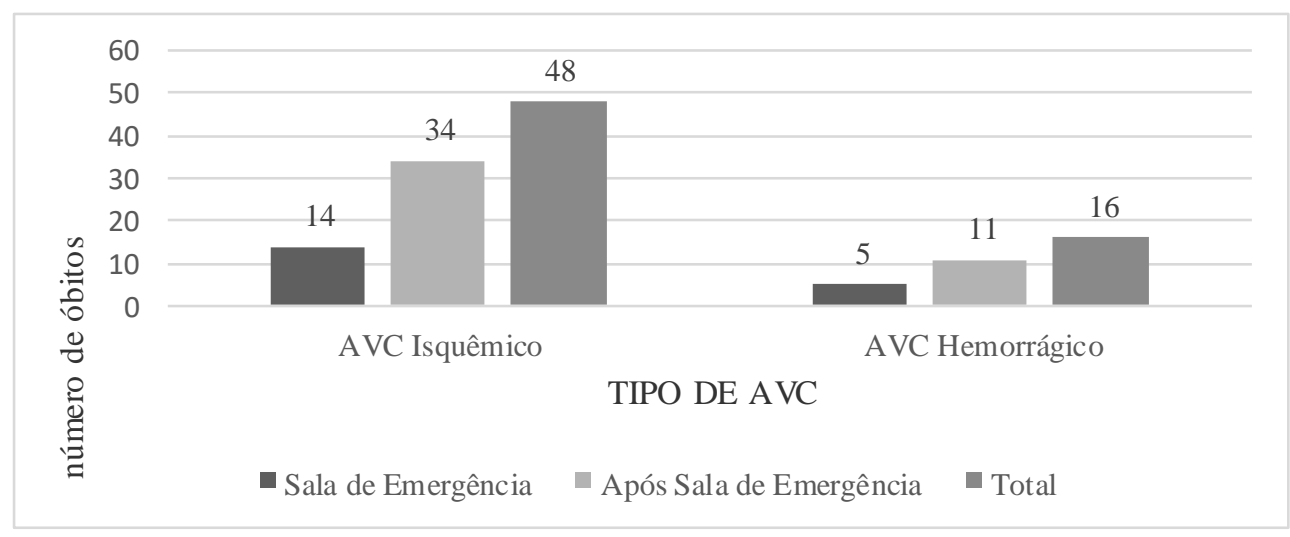

AVC: Acidente Vascular Cerebral. Fonte: Autoria Própria (2022).

Cordeiro e Lima (2017) realizaram uma revisão sistemática sobre a fisioterapia em unidades de urgência e emergência, onde, de acordo com os ensaios clínicos randomizados encontrados na pesquisa, a fisioterapia é de suma importância na urgência e emergência para a redução do quadro álgico do paciente, da necessidade de reinternação hospitalar e também melhora em relação a satisfação do paciente. Além disto, Santos et al. (2020) reinteram em seu estudo que, por conta de um elevado número de disfunções cardiopulmonares encontradas nas unidades de urgência e emergência, é necessária a atuação do fisioterapeuta para melhor manejo dos pacientes, também deixando claro que por ser uma unidade que serve como porta de entrada hospitalar, ocorre a admissão de diversas doenças, onde o fisioterapeuta pode atuar, assim, ressaltando a importância de protocolos motores associados a intervenções respiratórias, visto que em diversas doenças há comprometimento também da parte motora do paciente. Na Tabela 3 é possível verificar os dados sobre o atendimento fisioterapêutico nos pacientes com AVC. Houve uma média de 3,20 $( \pm 3,80)$ atendimentos fisioterapêuticos nestes indivíduos, variando de 0 a 23 
atendimentos. $84,95 \%$ dos pacientes foram atendidos pela equipe de fisioterapia durante o período de internamento na sala de emergência.

Tabela 3. Dados sobre o atendimento fisioterapêutico em pacientes com AVC na sala de emergência do Hospital Universitário do Oeste do Paraná $(\mathrm{n}=186)$.

\begin{tabular}{lll}
\hline Variável & $\mathbf{N}$ & $\%$ \\
\hline $\begin{array}{l}\text { Atendido pela fisioterapia durante internamento na sala de } \\
\text { emergência }\end{array}$ & & \\
Sim & 158 & $84,95 \%$ \\
Não & 28 & $15,05 \%$ \\
Condutas realizadas pela fisioterapia & & \\
Protocolo respiratório & 6 & $3,23 \%$ \\
Admissão e protocolo respiratório & 9 & $4,84 \%$ \\
Protocolo motor e respiratório & 100 & $53,79 \%$ \\
Admissão, protocolo motor e respiratório & 37 & $19,89 \%$ \\
Admissão & 6 & $3,23 \%$ \\
Não realizado & 28 & $15,05 \%$ \\
\hline
\end{tabular}

Fonte: Autoria Própria (2022).

Silva et al. (2014), encontraram uma média de $7( \pm 7,5)$ dias de acompanhamento da fisioterapia com os pacientes internados por AVC, onde o máximo foi de 43 dias e o mínimo de 1 dia, com uma média de $8( \pm 9,6)$ atendimentos fisioterapêuticos, sendo o mínimo de 1 e máximo de 62 atendimentos durante o internamento hospitalar total destes pacientes. O estudo analisou variáveis motoras funcionais, como o acometimento do membro superior e inferior, controle de tronco, equilíbrio estático e deambulação, onde foi realizada uma avaliação inicial (na admissão do paciente na unidade de AVC) e em uma avaliação final (correspondente ao dia de desfecho final do paciente), onde foi encontrado um aumento nos valores percentuais na maioria das variáveis motora-funcionais analisadas entre as avaliações. Sendo assim, o estudo traz que, de acordo com os resultados, uma equipe multiprofissional em uma unidade de AVC hospitalar, que apresente um eficaz serviço de reabilitação, pode auxiliar na redução ou até mesmo na prevenção de complicações decorrentes de lesões cerebrovasculares, principalmente em pacientes idosos, que são os principais acometidos pela doença.

Alamri et al. (2019) selecionaram 60 pacientes com diagnóstico de AVC confirmado, com idades entre 40 e 60 anos, que haviam sido admitidos nas UTIs gerais em Riade, Reino da Arábia Saudita dentro de 24 horas do início dos sintomas, no período de outubro e dezembro de 2016. Foi realizado um protocolo de mobilização precoce nestes pacientes, onde após as intervenções, verificaram uma melhora na força muscular, na função pulmonar e na qualidade de vida dos pacientes, sendo assim, a mobilização precoce é de extrema importância para pacientes de UTI e também naqueles hospitalizados em enfermarias, especialmente nos casos de AVC, pois tem um efeito positivo na capacidade funcional do paciente, além de auxiliar na redução do tempo de internamento hospitalar.

No presente estudo, os atendimentos incluíam protocolos motores, respiratórios (manobras e/ou exercícios de higiene brônquica; manobras e/ou exercícios para reexpansão pulmonar; aspiração de vias aéreas superiores e/ou tubo orotraqueal e/ou traqueostomia; manejo de ventilação mecânica invasiva), além da participação na admissão do paciente, juntamente com a equipe multidisciplinar, na chegada do paciente na Sala de Emergência. Na admissão, o atendimento fisioterapêutico incluiu a avaliação inicial do paciente; instalação, ajuste ou remoção de oxigenoterapia; preparação e acoplamento do paciente à ventilação mecânica invasiva e discussão com a equipe multiprofissional sobre os próximos passos a serem realizados com o 
paciente. Em relação aos pacientes que não receberam nenhum atendimento fisioterapêutico, nem mesmo os protocolos de admissão do paciente, foram todos relacionados a admissões no período noturno, e em seguida já eram transferidos para a UTI, ou enfermaria, ou para outra unidade hospitalar, e também, aqueles que eram encaminhados emergencialmente para o centro cirúrgico e logo em seguida para outros setores, não retornando à sala de emergência. Vale ressaltar que, durante o período de coleta dos dados do presente estudo, não havia atuação da equipe fisioterapêutica na sala de emergência no período noturno no HUOP.

\section{Conclusão}

O presente estudo vai de acordo com estudos atuais, onde encontra-se uma maior prevalência de casos de AVC do tipo isquêmico, além de maior prevalência no sexo masculino, em idades acima de 60 anos e brancos. Foi verificado um tempo médio de internamento de 2,43 ( $\pm 2,22)$ dias na sala de emergência, onde o mínimo foi de 1 dia e máximo de 18 dias. Quanto ao destino do paciente após o internamento no local, a maior prevalência é daqueles encaminhados para a enfermaria. Quanto ao desfecho, a maioria dos pacientes receberam alta hospitalar, ocorrendo apenas 64 óbitos. Em relação a fisioterapia, foram principalmente empregadas condutas motoras e respiratórias associadas, e apenas 15,05\% não receberam nenhum tipo de atendimento fisioterapêutico, a maioria internado no período noturno, em que não havia atuação da fisioterapia no setor, ou encaminhados para o centro cirúrgico logo após a admissão na sala de emergência.

Por se tratar de um hospital escola, os dados da atual pesquisa podem auxiliar na avaliação e tratamento da população estudada, além de ressaltar a necessidade e importância do profissional fisioterapeuta como parte da equipe multidisciplinar em uma unidade de urgência e emergência, especialmente nos casos de AVC.

Sugere-se para estudos futuros que sejam avaliados os efeitos das técnicas convencionais e não convencionais de fisioterapia respiratória e motora nos casos de AVC, assim auxiliando estes profissionais na prevenção e tratamento desta população, além de auxiliar cada vez mais na inserção de tal profissional dentro da equipe multidisciplinar.

\section{Referências}

Alamri, M. S., Waked, I. S., Amin, F. M., Al-Quliti, K. W., \& Manzar, M. D. (2019). Effectiveness of an early mobility protocol for stroke patients in Intensive Care Unit. Neurociences, 24(2), 81-88.

Alhazzani, A. A., Mahfouz, A. A., Abolyazid, A. Y., Awadala, N. J., Katramiz, K., Faraheen, A. \& Aftab, R. (2018). In Hospital Stroke Mortality: Rates and Determinants in Southwestern Saudi Arabia. International Journal of Environmental Research and Public Health, $15(5), 927$.

Almeida, I. C. N., Lima, G. M., Costa, L. A., Carneiro, L. M., Santos, M. I. G, Costa, L. R. N., \& Macêdo, R. C. (2017) Atuação da fisioterapia na urgência e emergência de um hospital referência em trauma e queimados de alta e média complexidade. Revista da Universidade Vale do Rio Verde, 15(1), 791-805.

Almeida, L. G., \& Vianna, J. B. M. (2018). Perfil epidemiológico dos pacientes internados por acidente vascular cerebral em um hospital de ensino. Revista Ciências em Saúde, 8(1), 12-17.

Aquino, A. C. (2019) Acidente Vascular Cerebral: epidemiologia e desfechos. (Trabalho de Conclusão de Curso (graduação)). Universidade Federal da Fronteira Sul, Passo Fundo, Brasil.

Barbosa, A. M. L., Pereira, C. C. M., Miranda, J. P. R., Rodrigues, J. H. L., Carvalho, J. R. O., \& Rodrigues, A. C. E. (2021). Perfil epidemiológico dos pacientes internados por Acidente Vascular Cerebral no nordeste do Brasil. Revista Eletrônica Acervo Saúde, 13(1), e5155-5155.

Baron, B. (2020). Pronto socorro do HUOP atende cerca de 200 pacientes por dia. https://www.unioeste.br/portal/central-de-noticias/50862-pronto-socorrodo-huop-atende-cerca-de-200-pacientes-por-dia.

Bensenor, I. M., Goulart, A. C., Szwarcwald, C. L., Vieira, M. L. F. P., Malta, D. C., \& Lotufo, P. A. (2015). Prevalência de acidente vascular cerebral e de incapacidade associada no Brasil: Pesquisa Nacional de Saúde - 2013. Arquivos Neuropsiquiatria, 73(9), 746-50.

Coelho, M. J. B. (2019). Perfil dos atendimentos infantis no pronto socorro do hospital universitário de vassouras. (Dissertação de mestrado em Ciências Aplicadas em Saúde). Universidade de Vassouras, Vassouras - Rio de Janeiro.

Cordeiro, A. L., vLima, T. G. (2017). Fisioterapia em unidades de emergência: uma revisão sistemática. Revista Pesquisa em Fisioterapia, 7, (2), 276-281.

Dabilgou, A. A., Dravé, A., Kyelem, J. M. A., Ouedraogo, S.; Napon, C., \& Kaboré, J. (2020). Frequency and Mortality Risk Factors of Acute Ischemic Stroke in Emergency Department in Burkina Faso. Stroke Research and Treatment, 2020, 1-7. 
Duarte, M. P., Rose, L. L. D., Pinheiro, E. M., Alves, I. K., Santos, A. A., Andrade, Á. D. B. \& Diniz, W. A. (2020). Influência da fisioterapia na reabilitação de pacientes submetidos a drenagem torácica em um hospital de urgência e emergência da amazônia legal. Revista Eletrônica Acervo Saúde, (45), e2959e2959.

Fernandes, T. G., Goulart, A. C., Santos, W. R., Jr., Alencar, A. P., Benseñor, I. M., \& Lotufo, P. A. (2012). Educational levels and the functional dependence of ischemic stroke survivors. Cadernos de Saúde Pública, 28(8), 1581-1590

Figueiredo, A. R. G., Pereira, A., \& Mateus, S. (2020). Acidente Vascular Cerebral isquêmico vs hemorrágico: taxa de sobrevivência. HIGEIA-Revista Científica da Escola Superior de Saúde Dr. Lopes Dias, 3(1), 35-45.

Fundação Instituto Brasileiro de Geografia e Estatistica (2013). Pesquisa Nacional de Saúde. http://www.sidra.ibge.gov.br/bda/ pesquisas/pns/Volume1.asp.

Lima, A. S. N., Fratelli, C. F., Freire, D. O., Ferreira, L. B., Souza, H. C., \& Silva, I. C. R. (2020). Associação do polimorfismo genético no gene BCL2 no Acidente Vascular Cerebral Hemorrágico/Aneurisma. Revista de Divulgação Cientifica Sena Aires, 9(1), 118-124.

Lima, C. M. G., Silva, H. P. W., Souza, P. A. S., Amaral, T. L. M., \& Prado, P. R. (2015). Caracteristicas epidemiológicas e clínicas dos pacientes acometidos por acidente vascular cerebral. Journal of the Health Sciences Institute, 33(1), 45-49.

Lopes, J. M., Sanchis, G. J. B., Medeiros, J. L. A., \& Dantas, F. G. (2016). Hospitalization for ischemic stroke in Brazil: an ecological study on the possible impact of Hiperdia. Revista Brasileira de Epidemiologia, 19(1), 122-134.

Moura, A., Carvalho, J. P. G., \& Silva, M. A. B. (2018). Urgência e emergência: conceitos e atualidades. Saúde \& Conhecimento-Jornal de Medicina Univag, $1,12-18$.

Mourão, A. M., Vicente, L. C. C., Chaves, T. S., Sant'Anna, R. V., Meira, F. C., Xavier, R. M. B. \& Teixeira, A. L. (2017). Perfil dos pacientes com diagnóstico de AVC atendidos em um hospital de Minas Gerais credenciado na linha de cuidados. Revista Brasileira de Neurologia, 53(4), 12-16.

Radanovic, M. (2000). Características do atendimento de pacientes com acidente vascular cerebral em hospital secundário. Arquivos de neuro-psiquiatria, 58(1), 99-106.

Rebouças, E. S., Braga, A. M., Sarmento, R. M., Marques, R. C. P., \& Rebouças, P. P. F. (2017). Level Set Based on Brain Radiological Densities for Stroke Segmentation in CT Images. 2017 IEEE 30th International Symposium on Computer-Based Medical Systems (CBMS), 391-396.

Rodrigues, M. S., Santana, L. F., \& Galvão, I. M. (2017). Fatores de risco modificáveis e não modificáveis do AVC isquêmico: uma abordagem descritiva. Revista de Medicina, 96(3), 187-192.

Rolim, C. L. R. C., \& Martins, M. (2011). Qualidade do cuidado ao Acidente Vascular Isquêmico no SUS. Cadernos de Saúde Pública [online], 27(11), 21062116.

Sá, B. P., Grave, M. T. Q., \& Périco, E. (2014). Perfil de pacientes internados por Acidente Vascular Cerebral em hospital do Vale do Taquari/RS. Revista Neurociências, 22(3), 381-387.

Santana, N. M., Figueiredo, F. W. S., Lucena, D. M. M., Soares, F. M., Adami, F., Cardoso, L. C. P., \& Correa, J. A. (2018). The burden of stroke in Brazil in 2016: an analysis of the Global Burden of Disease study findings. BMC research notes, 11(1), 1-5.

Santos, K. R., Luquini, V. C., \& Fagundes, T. R. (2020). Epidemiologia dos óbitos relacionados a Acidente Vascular Cerebral ocorridos no Estado do Paraná: uma comparação entre os anos de 2008 e 2018. Research, Society and Development, 9(11), e389119527-e389119527.

Santos, L. B., \& Waters, C. (2020). Perfil epidemiológico dos pacientes acometidos por Acidente Vascular Cerebral: revisão integrativa. Brazilian Journal of Development, 6(1), 2749-2775.

Santos, P. R., Nepomuceno, P., Reuter, É. M., \& Carvalho, L. L. (2020). Percepção da equipe multiprofissional sobre o fisioterapeuta na emergência de um hospital do interior do Rio Grande do Sul. Revista Fisioterapia e Pesquisa, 27(2), 147-154.

Schmidt, M. H., Selau, C. M., Soares, P. S., Franchi, E. F., Piber, V. D., \& Quatrin, L. B. (2019). Acidente Vascular Cerebral e diferentes limitações: uma análise interdisciplinar. Arquivos de Ciências e Saúde da UNIPAR, 23(2), 139-144.

Silva, C. C. M., \& Santos, I. M. (2019). A importância da fisioterapia no setor de urgência e emergência: uma revisão de literatura. Brazilian Journal of Development, 5(10), 18335-18343.

Silva, M. C. L., Polese, J. C., Starling, J. M. P., \& Pereira, L. S. M. (2014). Caracterização clínica e motora-funcional de idosos hospitalizados pós-Acidente Vascular Cerebral. Revista Neurociências, 22(3), 337-343.

Silva, P. L. N., Santos, L. S., Ribeira, D. D. D., Guimarães, H. D. G., Esteves, K. A., Alves, E. C. S., \& Marinho, H. V. R. (2018). Análise da prevalência de acidente vascular encefálico em pacientes assistidos por uma instituição hospitalar. Journal of Management \& Primary Health Care, 9, 1-15.

Silva, R. G., Cobo, D. L., Foss, M. H. D., Vale, M. E., \& Cavenaghi, S. (2021). Perfil epidemiológico da unidade de AVC em um hospital de ensino. Brazilian Journal of Health Review, 4(5), 22023-22030.

Sociedade Brasileira de Doenças Cerebrovasculares. (2001). Primeiro consenso brasileiro do tratamento da fase aguda do acidente vascular cerebral. Arquivos de Neuro-Psiquiatria, 59(4), 972-980.

Universidade Estadual do Oeste do Paraná (2020, 30 de março). Dados Estatísticos. https://www.unioeste.br/portal/institucional-huop/dados-estatisticos.

Vieira, S., \& Hossne, W. S. (2021). Metodologia científica para a área da saúde (3a ed.). Guanabara Koogan.

World Health Organization. (2018). Health statistics and information systems - projections of mortality and causes of death, 2015 and 2030 . WHO. 